Original Article

\title{
Combining rescanning and gating for a time-efficient treatment of mobile tumors using pencil beam scanning proton therapy
}

\author{
Pauline Gut $^{\mathrm{a}, \mathrm{b}}$, Miriam Krieger ${ }^{\mathrm{a}, \mathrm{b}}$, Tony Lomax ${ }^{\mathrm{a}, \mathrm{b}}$, Damien C. Weber ${ }^{\mathrm{b}, \mathrm{c}, \mathrm{d}}$, Jan Hrbacek ${ }^{\mathrm{b}, *}$

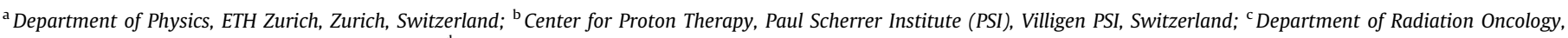
University Hospital of Zurich, Zurich, Switzerland; ${ }^{\mathrm{d}}$ Department of Radiation Oncology, Inselspital, Bern University Hospital, University of Bern, Switzerland

\section{A R T I C L E I N F O}

\section{Article history:}

Received 2 November 2020

Received in revised form 4 March 2021

Accepted 31 March 2021

Available online 9 April 2021

\section{Keywords:}

4D proton therapy

Time efficiency

Tumor coverage

Intrafractional motion

Pencil beam scanning

Volumetric rescanning

Respiratory gating

\begin{abstract}
A B S T R A C T
Background and purpose: Respiratory motion during proton therapy can severely degrade dose distributions, particularly due to interplay effects when using pencil beam scanning. Combined rescanning and gating treatments for moving tumors mitigates dose degradation, but at the cost of increased treatment delivery time. The objective of this study was to identify the time efficiency of these dose degradationmotion mitigation strategies for different range of motions.

Materials and methods: Seventeen patients with thoracic or abdominal tumors were studied. Tumor motion amplitudes ranged from 2-30 mm. Deliveries using different combinations of rescanning and gating were simulated with a dense dose spot grid $\left(4 \times 4 \times 2.5 \mathrm{~mm}^{3}\right)$ for all patients and a sparse dose spot grid $\left(8 \times 8 \times 5 \mathrm{~mm}^{3}\right)$ for six patients with larger tumor movements $(>8 \mathrm{~mm})$. The resulting plans were evaluated in terms of CTV coverage and time efficiency.

Results: Based on the studied patient cohort, it has been shown that for amplitudes up to $5 \mathrm{~mm}$, no motion mitigation is required with a dense spot grid. For amplitudes between 5 and $10 \mathrm{~mm}$, volumetric rescanning should be applied while maintaining a $100 \%$ duty cycle when using a dense spot grid. Although gating could be envisaged to reduce the target volume for intermediate motion, it has been shown that the dose to normal tissues would only be reduced marginally. Moreover, the treatment time would increase. Finally, for larger motion amplitudes, both volumetric rescanning and respiratory gating should be applied with both spot grids. In addition, it has been shown that a dense spot grid delivers better CTV dose coverage than a sparse dose grid.

Conclusion: Volumetric rescanning and/or respiratory gating can be used in order to effectively and efficiently mitigate dose degradation due to tumor movement.
\end{abstract}

( $) 2021$ Published by Elsevier B.V. Radiotherapy and Oncology 160 (2021) 82-89
Proton therapy has become a technique that has spread worldwide with the construction of many proton therapy centers, most of which deliver pencil beam scanning (PBS). However, the major issue with $\mathrm{PBS}$ proton therapy is the handling of intrafractional motion. The strong range dependence and interplay effect of PBS lead to significant degradation of the dose distribution in the presence of intrafractional motion. This results in inhomogeneous dose distributions with local under- and over-dosage in the target and over-dosage in the surrounding normal tissues [1-3]. Various motion mitigation techniques have been developed to mitigate motion effects for PBS based treatments and which maintain target conformity of the delivered dose distributions [4-7,2].

Breath-hold [8] and beam gating [9-11] techniques can both reduce the interplay effect by controlling the motion amplitude.

\footnotetext{
* Corresponding author at: Paul Scherrer Institute, Forschungsstrasse 111, 5232 Villigen PSI, Switzerland.

E-mail address: jan.hrbacek@psi.ch (J. Hrbacek).
}

However, firstly, not every patient is able to hold his/her breath during treatment. Secondly, although it has been shown that gating becomes important when tumor motion is large [12], gating increases considerably the treatment time and residual interplay effects may still need to be addressed. Another technique, socalled beam tracking, would have the potential to adapt the scanned beam to target motion laterally as well as longitudinally in depth [13-15], but has not yet been used clinically. Alternatively, rescanning [1] has the potential to average out the local over- and under-dosage by delivering the fraction dose in repeated scans. Several approaches to rescanning have been proposed. Previous studies have shown its impact on the final dose distribution, such as the quantification of the interplay effects as well as plan robustness, but it's effectiveness can be machine specific [16]. On the other hand, although this technique has proven its effectiveness for mobile tumors in many previous studies [17-21], the target volume has to be extended to cover the whole motion of the 
tumour. As a consequence, the irradiated volume of healthy tissue becomes larger as motion amplitudes increase [22,20].

Previously at PSI, all patients were treated only with volumetric rescanning, restricting the treatments to moderately moving targets (motions $<8 \mathrm{~mm}$ ). The introduction of an optical tracking system into our clinic opened the possibility of gated delivery [23], with the effectiveness of combined gating and rescanning for treating mobile targets with proton PBS being demonstrated in previous studies [10]. However, both techniques increase considerably the time needed for the delivery, particularly with gating. Thus, this study aims to identify which combinations of gating and rescanning lead to clinically acceptable results and, among those, to identify the most time-efficient combinations.

This study is a systematic analysis of the effectiveness and efficiency of clinically applied motion mitigation strategies and may serve as a clinical guideline for future treatments of mobile tumors.

\section{Materials and methods}

\section{Patient cohort}

In order to simulate $4 \mathrm{D}$ treatments, available patient data sets with moving tumors were investigated. A total of 17 patients were studied, including different anatomical tumor sites such as thorax and abdomen (Table 1). Patients 1-5, 8, 14-17, have been previously treated at PSI, whereas patients 6-7, 9-13, have not been treated at PSI, but were considered as test cases. The clinical target volume (CTV) of each patient can be found in Table 1. Moreover, in this study, the original treatment prescription was kept, but only one fraction was analysed. The dose was chosen based on the original treatment prescription for PSI patients. They varied between 0.3 and $5 \mathrm{~Gy} /$ fraction, see Table 1. For non-PSI patients, we chose a dose of $2 \mathrm{~Gy} /$ fraction (not the prescription), because this data was not provided.

\section{Motion extraction}

Every patient had a 4DCT in order to take into consideration the tumor motion, and provides time-resolved density information for both treatment planning and delivery [24-28]. To quantify the motion during a breathing cycle, deformable image registration (DIR) has been used [29-34] and perfectly regular breathing assumed for each patient. In this way, CTV motion through a respiratory cycle could be extracted for every patient.

The CTV motion in this study was defined as the median of the peak-to-peak displacement of the centre of each voxel contained in the CTV throughout all phases. From this, the motion range of the CTV over the entire breathing cycle in the superior-inferior (SI) direction, anterior-posterior (AP) direction, and left-right (LR) direction have been extracted. The CTV movement in the SI direction within the entire breathing cycle was the largest motion for every patient, with unmitigated CTV motion in the SI direction for all patients covering a range from $1.87 \mathrm{~mm}$ to $30.07 \mathrm{~mm}$ (Table 1). Breathing periods extracted from the 4DCT temporal resolution can be found in Table 1 for all patients.

III. Motion mitigation techniques

Both respiratory gating and hybrid volumetric scaled rescanning techniques have been considered in this study as motion mitigation strategies.

\section{A. Respiratory gating}

A gating window was defined as the number of breathing phases in the 4DCT. As such, the gating window definition is not amplitude-based. Since exhalation is the most reproducible respiratory state, the exhale phase has been selected to treat tumors $[35,36]$ and a gating window defined as the number of phases around the full exhalation phase. Hence, each gating window determined a duty cycle (DC), simply given by:

$$
D C=\frac{\text { Number of phases within the gating window }}{\text { Total number of phases within a respiratory cycle }}
$$

For our study, different DCs were investigated to attenuate residual tumor movement, that is, the movement of the target volume within the gating window. The following four DCs were chosen for every patient: a 100\% DC, which included all breathing phases (i.e. no gating) a $60 \%$ DC, a $50 \%$ DC and a $40 \%$ DC.

\section{B. Volumetric rescanning}

In clinical practice, our institution uses volumetric, hybrid scaled rescanning, enabled by fast energy changes of the gantry beamline $[37,1,3,38,17,39]$. For this, the dose of every spot is divided by the number of rescans (NR), referred to as scaled repainting. However, when spots are too low-weighted, these cannot be sub-divided. As a consequence, if a spot cannot be divided by the nominal number of rescans (NR), the number of spot-specific rescans will be reduced as necessary. Thus, some spots with very low weights will be delivered only once. Hybrid scaled volumetric rescanning with NRs from 1-9 have been assessed for all patients.

Table 1

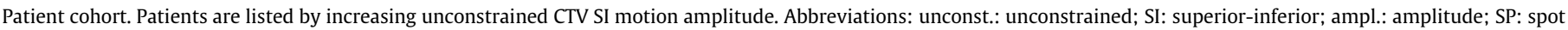
grid.

\begin{tabular}{|c|c|c|c|c|c|c|c|}
\hline $\begin{array}{l}\text { Patient } \\
\text { ID }\end{array}$ & $\begin{array}{l}\text { Anatomical tumor } \\
\text { site }\end{array}$ & $\begin{array}{l}\text { CTV volume } \\
\left(\mathrm{cm}^{3}\right)\end{array}$ & $\begin{array}{l}\text { Unconst. SI motion ampl. } \\
(\mathrm{mm})\end{array}$ & $\begin{array}{l}\text { Fraction dose } \\
\text { (GyRBE) }\end{array}$ & $\begin{array}{l}\text { Breathing period } \\
(\mathrm{s})\end{array}$ & $\begin{array}{l}4 \times 4 \times 2.5 \mathrm{~mm}^{3} \\
\text { SP }\end{array}$ & $\begin{array}{l}8 \times 8 \times 5 \mathrm{~mm}^{3} \\
\mathrm{SP}\end{array}$ \\
\hline 1 & Lung & 129 & 1.87 & 2 & 3.05 & v & \\
\hline 2 & Thoracic wall & 774 & 2 & 1.8 & 3.05 & レ & \\
\hline 3 & Lung & 163 & 2.45 & 0.3 & 6.4 & r & \\
\hline 4 & Thoracic wall & 167 & 2.45 & 1.8 & 6.4 & $\nu$ & \\
\hline 5 & Lung & 1228 & 4.53 & 1.5 & 6.4 & v & \\
\hline 6 & Thoracic wall & 74 & 5.57 & 2 & 8.3 & r & \\
\hline 7 & Thoracic wall & 59 & 5.69 & 2 & 10 & r & \\
\hline 8 & Liver & 42 & 6.59 & 5 & 5.84 & $\nu$ & \\
\hline 9 & Thoracic wall & 24 & 6.64 & 2 & 4.76 & レ & \\
\hline 10 & Thoracic wall & 56 & 7.73 & 2 & 7.69 & r & \\
\hline 11 & Thoracic wall & 67 & 8.44 & 2 & 7.14 & $\nu$ & $\nu$ \\
\hline 12 & Thoracic wall & 107 & 11.36 & 2 & 6.25 & v & 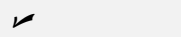 \\
\hline 13 & Thoracic wall & 6 & 12.14 & 2 & 8.3 & レ & v \\
\hline 14 & Abdomen & 218 & 12.95 & 2.5 & 5.06 & $\nu$ & $\nu$ \\
\hline 15 & Liver & 47 & 14.76 & 5 & 4.92 & $\nu$ & r \\
\hline 16 & Liver & 75 & 21.79 & 5 & 4.92 & v & $\nu$ \\
\hline 17 & Liver & 21 & 30.07 & 5 & 5.84 & $\nu$ & \\
\hline
\end{tabular}




\section{Machine characteristics}

In this paragraph, some important features of the proton machine are presented. Detailed machine characteristics can be found in [3,37,40-42]. To allow a fast two-dimensional scanning, two sweeper magnets ( $\mathrm{U}$ and $\mathrm{T}$ direction) are installed on the gantry. The scanning speeds are of $0.5 \mathrm{~cm} /$ $\mathrm{ms}$ in U-direction (dispersive direction) and of $2 \mathrm{~cm} / \mathrm{ms}$ in Tdirection (transverse to the magnets). The energy variation is performed right after the cyclotron using a degrader ( $\mathrm{S}$ axis). A typical beam energy change of $3 \mathrm{MeV}$ for typical range steps of $5 \mathrm{~mm}$ water equivalent can be made within $80 \mathrm{~ms}$.

The energy can be changed in the range between $70 \mathrm{MeV}$ and $230 \mathrm{MeV}$. The components in the nozzle are designed to have as little material as possible in the beam path. This maintains a small spot size, $\sigma=3-4 \mathrm{~mm}$, at all energies.

\section{Treatment planning}

A geometrical internal target volume (gITV) [22] was generated for every patient in order to consider anatomical changes due to breathing during both treatment planning and treatment delivery [43-45]. The gITV is defined as the volume including all clinical target volume (CTV) positions at all respiratory phases during the respiratory cycle when the beam is on. Finally, to account for uncertainties during treatment delivery such as patient positioning errors, a margin of $7 \mathrm{~mm}$ has been added to the gITV for the treatment planning process, defining the planning target volume (PTV).

For treatment planning, a synthetic static CT has been created from a $4 \mathrm{DCT}$ for the optimization process, in order to limit plan degradation due to respiratory motion [46,45].

In a first step, the maximum intensity of all breathing phases per voxel in the gITV was calculated, referred to as an intensity filling overwrite [46]. This step was necessary to position the Bragg peaks in low-density tissues during the treatment planning procedure. Then, in a second step, and in order not to plan the treatment on a specific respiratory start phase, voxel-wise Hounsfield units outside the gITV were averaged over all breathing phases. As a result, a synthetic static CT has been created from a $4 \mathrm{DCT}$ for the optimization process, limiting plan degradation due to respiratory motion $[46,45]$.

Based on this synthetic static CT, multiple field clinical treatment plans were created for each patient. The treatment fields were selected so that they best cover the tumor while avoiding neighbouring critical structures and organs [47]. Each field was individually optimized through a single field uniform dose (SFUD) optimization, so that each field delivered homogeneous target dose coverage [47].

\section{4D dose calculation}

Treatment deliveries were simulated using the PSI in-house 4D dose calculation (4DDC) engine described by Boye et al. [48] and Krieger et al. [49]. The 4DDC is able to show the motion effects on the static dose distributions. The PSI inhouse made 4DDC algorithm is based on both the ray casting algorithm and on a deformable dose grid algorithm. The idea of this PSI 4DDC is to calculate the dose of each pencil beam based on an individual time stamp while taking into account deformations and displacements over the breathing cycle of the 4DCT. The 4DDC uses the motion vector field extracted from the $4 \mathrm{DCT}$ to deform the dose calculation grid as function of time. Based on 4DCT information, water-equivalent path lengths and density information are adapted for every point in time.

All plans have been calculated with a spot grid size of $4 \times 4 \times 2.5 \mathrm{~mm}^{3}$ (2.5 $\mathrm{mm}$ is the energy layer spacing in water) as used clinically in our institution [41]. In addition, patients 11-16 (with larger tumor motions) have also been calculated with a sparser spot grid, $8 \times 8 \times 5 \mathrm{~mm}^{3}$, which may be more representative for other institutions (Table 1 ). The $4 \times 4 \times 2$. $5 \mathrm{~mm}^{3}$ spot grid and the $8 \times 8 \times 5 \mathrm{~mm}^{3}$ spot grid have spots spacing of $4 \times 4 \mathrm{~mm}^{2}$ and $8 \times 8 \mathrm{~mm}^{2}$, respectively, and energy layers spacing of $2.5 \mathrm{~mm}$ and $5 \mathrm{~mm}$ water equivalent, respectively.

For each defined field in a patient's treatment plan, a delivery start phase needs to be chosen. However, since the start of the delivery is not synchronized with the respiration of the patient, all possible combinations of starting phases, within the respective gating window as defined by the number of phases of the 4DCT contained with the gating window, were simulated. Hence, for every patient and for each spot grid, the total number of different dose distributions obtained for each DC combined for only NR factor, is given by:

(number of breathing phases in the gating window) ${ }^{\text {number of fields }}$

As a result, with an average of 2.4 fields for each treatment plan, more than 7000 dose distributions have been calculated in total (Eq. (1), Table 2). In combination with the different phases per $4 \mathrm{D}$ scenario, this has resulted in more than 300 '000 dose distributions having been calculated in total.

\section{Evaluation}

CTV dose coverage has been assessed by the percentage of volume receiving at least $95 \%$ of dose (V95\%). Once all dose distributions have been calculated, CTV dose coverage was analysed for every combination of NR and DC for all patients. For each patient, many different dose distributions have been obtained for one DC combined with one possible NR, due to combinations of all respiratory start phases for all fields (Table 2). Therewith, the distribution of all V95\% values can be visualized as a single box in a boxplot. For every patient, up to 9 rescans were applied for each DC. Hence, for each DC, there were 9 different combinations with volumetric rescanning. For each combination there are different respiratory start phases for all fields. In that respect, each combination can be visualised as a single box in a boxplot. Thus, 9 boxes per DC were represented in each boxplot - a total of $36((9$ boxes per DC $) *(4 D C))$ boxes for each patient.

To assess the quality of the patient's treatment plan (TP) under conditions of motion, only plans meeting the following condition were considered: The 25th percentile of the V95\% must be $\geqslant 95 \%$ for a particular combination of rescanning and gating. The 25 th percentile (1st quartile) was chosen instead of the minimum value, as only one bad combination could already exclude the plan, while the others could be very good combinations. In addition, it is clinically reasonable to be above $95 \%$. However, not all acceptable patient treatment plans are also time efficient. Due to the repetitive beam interruptions when gating is applied, the treatment time increases considerably. Consequently, we can define this increase of time compared to a non-mitigated delivery as the gating time penalty, $t_{G}$. In our case, $t_{G}$, is not patient-specific, but simply given by a factor of $100 / D C$, where $D C$ is the duty cycle considered (Fig. 1). In contrast, as illustrated in Fig. 1, the hybrid scaled volumetric rescanning time penalty, $t_{R}$, is patient-specific. Thus, for a particular combination of DC and number of rescanning (NR) for a specific patient, the total time penalty TTP for delivering such a combination is simply given by:

$$
T T P=t_{G} \cdot t_{R}
$$

Therefore, from all acceptable patient treatment plans, only those that were most time efficient were considered clinically feasible treatment plans. 
Table 2

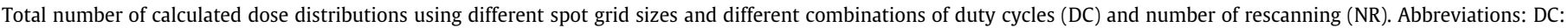
duty cycle; NR: number of rescanning.

\begin{tabular}{|c|c|c|c|c|c|c|}
\hline Spot grid & DC $=100 \%$ & $\mathrm{DC}=60 \%$ & $\mathrm{DC}=50 \%$ & $\mathrm{DC}=40 \%$ & Total with $1 \mathrm{NR}$ & Total with up to $9 \mathrm{NR}$ \\
\hline $4 \times 4 \times 2.5 \mathrm{~mm}^{3}$ & 3'277 & 992 & 612 & 335 & $5 ' 215$ & $234^{\prime} 675$ \\
\hline \multirow[t]{2}{*}{$8 \times 8 \times 5 \mathrm{~mm}^{3}$} & $1 ' 243$ & 364 & 226 & 125 & 1'958 & $88^{\prime} 130$ \\
\hline & & & & & $7 ’ 173$ & $322 ’ 805$ \\
\hline
\end{tabular}
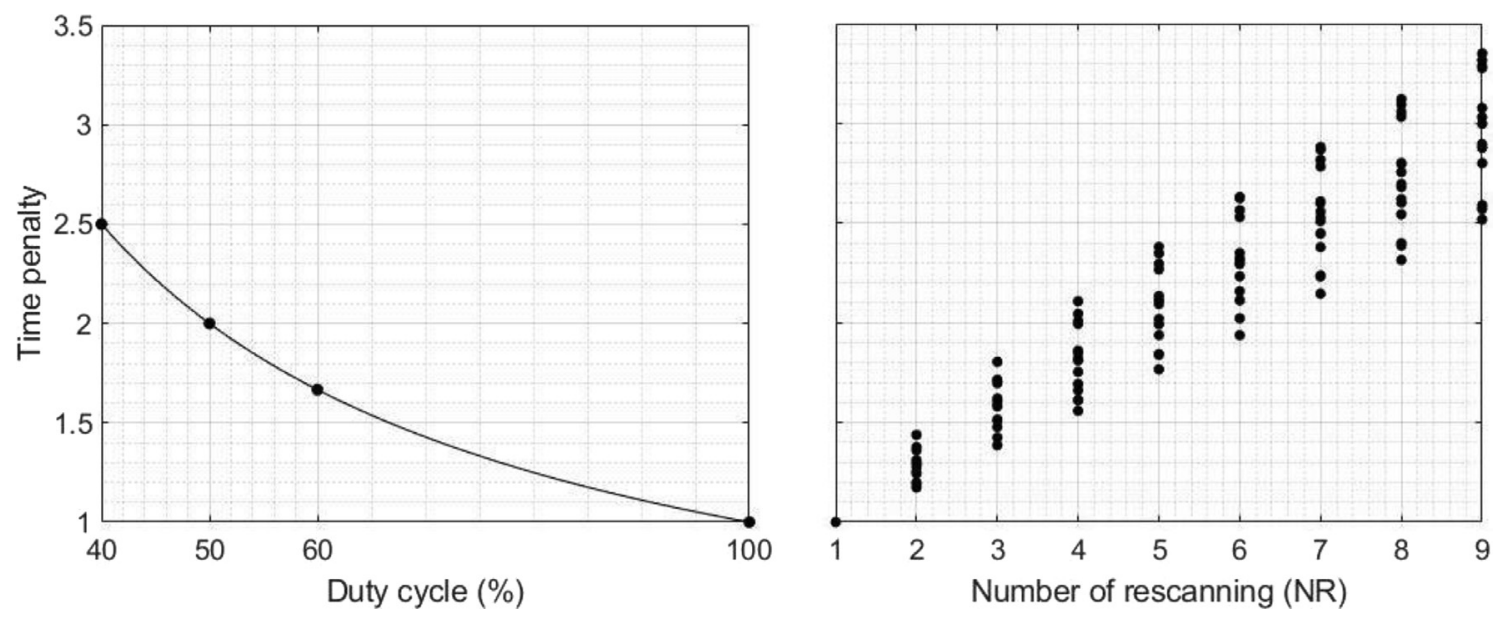

Fig. 1. Left: Gating time penalty, $t_{G}$. Right: Hybrid volumetric rescanning time penalty, $t_{R}$ for every patient.

\section{Results}

In the following, the results using a dense spot grid of $4 \times 4 \times$ $2.5 \mathrm{~mm}^{3}$ and a sparse spot grid of $8 \times 8 \times 5 \mathrm{~mm}^{3}$ will be illustrated for a patient (Patient ID: 16) with a large CTV SI motion (22 $\mathrm{mm}$ ) without gating ( $D C=100 \%$ ). Fig. 2 shows this patient's boxplot when using the dense spot grid. Each box is positioned on the $\mathrm{x}$ axis according to the time needed to deliver it, with each DC having a specific color: green for DC $=100 \%$, yellow for DC $=60 \%$, blue for $D C=50 \%$ and pink for DC $=40 \%$. Each combination of DC and NR is represented as a single box in a boxplot. As time increases, boxes of the same color represent an increasing NR from left to right. Furthermore, for each DC, the V95\% value was extracted for the static case. Since the V95\% values for each DC in the static case were all similar, the mean value was calculated and plotted as a solid black line in the boxplot. As illustrated in Fig. 2, it can be noticed that CTV dose coverage may decrease with increasing number of volumetric rescans due to interplay effects. If the number of rescans is increased, the time per rescan decreases because there is less dose delivered with each rescan. This means that the regularity between the rescan and breathing changes with the number of rescans. If every rescan starts in the same respiratory phase, the advantage of rescanning is lost and such interplay effects can occur. The same effects were observed in [10].

From all the boxplots of each patient, the CTV dose coverage was analysed with the following criterion: if the first quartile of a box was greater than or equal to $95 \%$ for V95\%, then the plan, consisting of a specific combination of DC and NR, was considered an acceptable treatment plan. The combinations meeting this criterion for patient number 16 are illustrated in the left panel of Fig. 3a for a spot grid size of $4 \times 4 \times 2.5 \mathrm{~mm}^{3}$. The right panel of Fig. 3a illustrates the same information for the larger spot spacing $\left(8 \times 8 \times 5 \mathrm{~mm}^{3}\right)$. It is seen that the overall delivery time is decreased by using a larger spot spacing. Nevertheless, less combinations of rescanning and gating are clinically acceptable (V95\% $\geqslant 95 \%$ ). All the results are summarized in Fig. $3 \mathrm{~b}$.
For every patient however, not all plans which meet the V95\% $\geqslant 95 \%$ condition are efficient in terms of time (Fig. 3a left). In order to maintain a certain clinical workflow, and to avoid organ drift and patient movements over the fraction, only the most time efficient plans with V95\% $\geqslant 95 \%$ were considered as clinically possible treatment plans. The most clinically feasible plan for each patient was then defined as the plan with the lowest delivery time that provided a target coverage of $\mathrm{V} 95 \% \geqslant 95 \%$. These are presented in Table 3 with a spot grid size of $4 \times 4 \times 2.5 \mathrm{~mm}^{3}$ for all patients (1-17) and with a spot grid size of $8 \times 8 \times 5 \mathrm{~mm}^{3}$ for patients 11-16.

In addition, the mean and maximum dose to the organ at risks (OARs) have been investigated for the two different spot grids. Regarding the mean dose reduction compared to the $100 \%$ DC with the clinical (dense) spot grid, a reduction of up to $3 \%$ could be achieved with a $40 \%$ DC for tumor motions below $5 \mathrm{~mm}$, a reduction up to $4 \%$ with a $40 \%$ DC for intermediate tumor motions (5$10 \mathrm{~mm}$ ), and a reduction up to $12 \%$ with a $40 \%$ DC for large tumor motions. With the sparser dose grid, dose to OARs can be reduced up to $5 \%$ for a $100 \%$ DC for large tumor motions. Regarding the maximum dose reduction compared to the $100 \%$ DC with the clinical spot grid, a reduction up to $4 \%$ to OARs could be achieved with a $40 \%$ DC for tumor motions below $5 \mathrm{~mm}$, a reduction up to $12 \%$ with a $40 \%$ DC for intermediate tumor motions (5-10 $\mathrm{mm})$, and a reduction up to $15 \%$ with a $40 \%$ DC for large tumor motions. For more details, please refer to the supplementary materials.

\section{Discussion}

In this study, we systematically investigated both the effectiveness of combined gating and rescanning for treating moving targets with PBS (efficacy) and the efficiency in terms of treatment delivery time. We did this comprehensive investigation with 17 very different real patients, we used the original clinical prescriptions, we looked at different tumor sites (such as lung, liver, pancreas), and we looked at a very wide unconstrained motion 

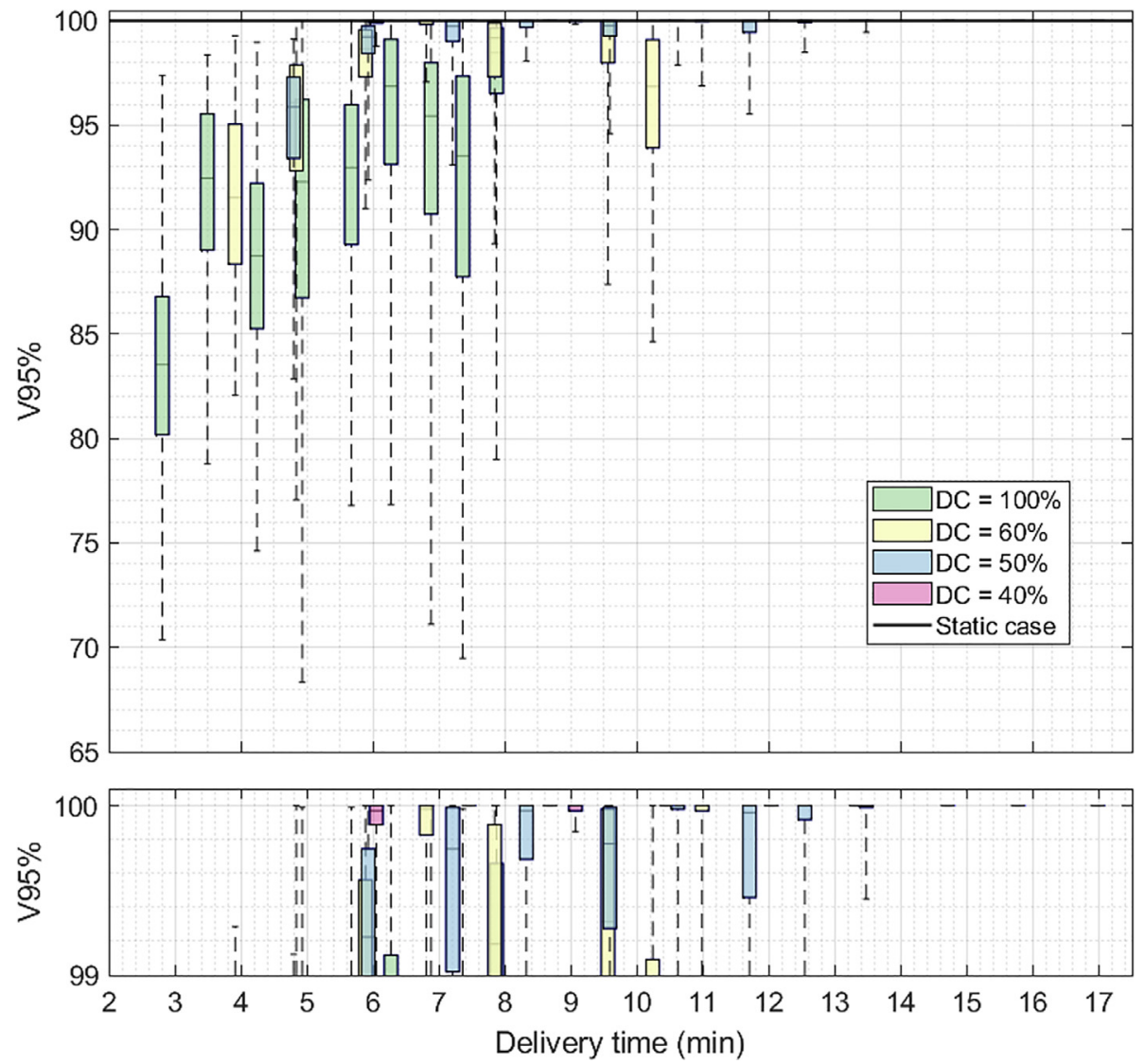

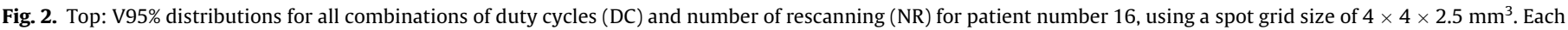

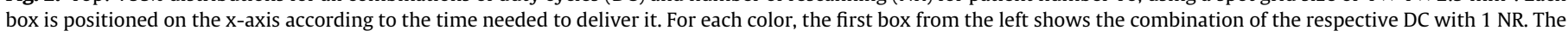

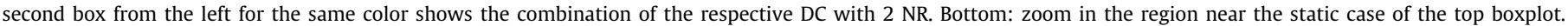
Abbreviation: DC: duty cycle.

amplitude interval. This study is a systematic analysis of the effectiveness and efficiency of clinically applied motion mitigation strategies and may serve as a clinical guideline for future treatments of mobile tumors.

The objective of this work was to identify the time efficiency of combined rescanning and gating treatments of moving tumors with proton pencil beam scanning in order to attenuate dose degradation due to tumor movement. For this purpose, treatment plans have been calculated with a clinical spot grid for seventeen patients (1-17 in Table 1), and, in addition, with a sparser dose grid for six patients (11-16 in Table 1) whose CTV SI motions were almost always the largest motions in the full duty cycle (DC $=100 \%$ ). Each plan has been analysed in terms of CTV dose coverage and delivery time efficiency. The plans meeting the condition V95\% $\geqslant 95 \%$ where considered clinically acceptable, and among those, the most time efficient where identified. The time efficiency of a treatment plan is clinically important to avoid organ drift and patient movements over a fraction, as well as for the patient comfort. Indeed, this was the main purpose of this study as the effectiveness of combined rescanning and gating treatments for moving tumors with proton spot scanning has been demonstrated in previous studies [10].

In clinical practice, $\mathrm{V} 95 \% \geqslant 95 \%$ is a common requirement to accept a treatment plan. Dose degradation within this criterion is clinically acceptable in clinical practice. During this study, it was seen that the better the V95\%, the better the homogeneity of the dose. However, unlike the V95\%, there is no clear acceptance measure for the dose homogeneity within the target. Moreover, even without motion (static case), the homogeneity in terms of D5\%-
D95\% was very different from one patient to another, making it difficult to define a threshold value. The D5\%-D95\% parameter heavily depends on the patient geometry and the tissue homogeneity. Therefore, $\mathrm{V} 95 \% \geqslant 95 \%$ was the only quantifiable criterion taken into account in deciding whether the plan was clinically acceptable. The visual comparison between the V95\% and the D5\%D95\% (see supplementary material for patient number 16) revealed the same patterns as a function of the number of rescans and the gating window. D5\%-D95\% implicitly also contains both cold and hot spots, which can occur under motion conditions. Furthermore, both the results for the D2\% as well as the D5\%-D95\% revealed the same patterns as the V95\%.

Based on the obtained gating and rescanning time penalties, it has been shown that gating penalizes more time than rescanning for small and intermediate motions and vice versa for large motions. Therefore, based on the studied patient cohort, and using the clinical spot grid size of $4 \times 4 \times 2.5 \mathrm{~mm}^{3}$, we would advise that for tumor motions smaller than $5 \mathrm{~mm}$ no motion mitigation is required in order to preserve both time efficiency and acceptable treatment quality. For intermediate movements (5-10 mm), fewer number of rescans should be applied while maintaining a $100 \%$ DC with the clinical spot grid. Although a $100 \%$ DC should be maintained to achieve more time efficient plans, gating could be envisaged to reduce the target volume. Nonetheless, it has been shown that respiratory gating does not necessarily reduce dose to normal tissues, or only marginally, for intermediate motions. Finally, for large to extremely large movements $(10-30 \mathrm{~mm})$, both gating and rescanning should be applied with both spot grids. Consequently, by applying gating, the dose to normal tissues could be 


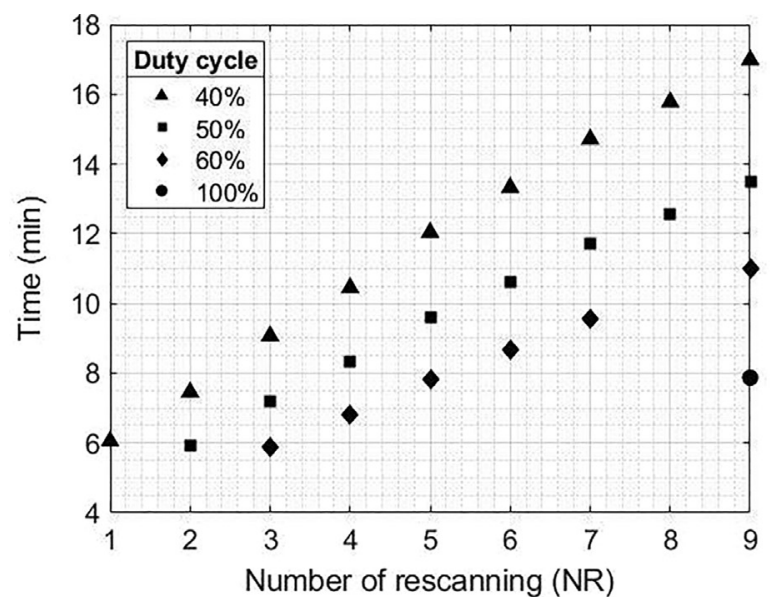

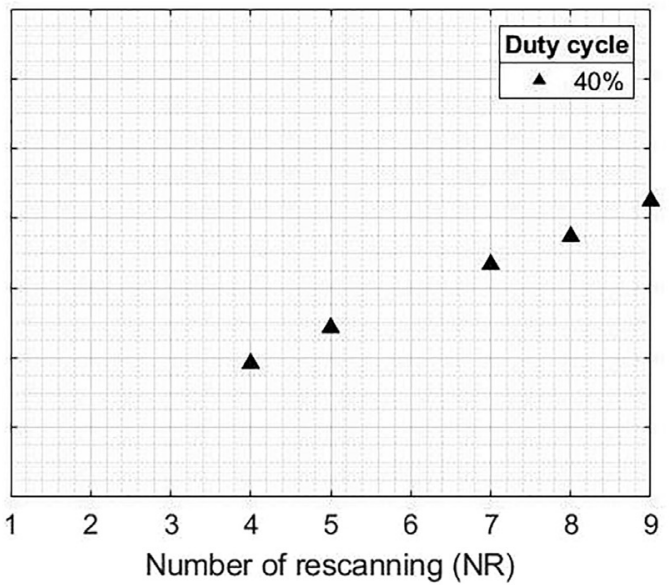

Number of rescanning (NR)

(a)
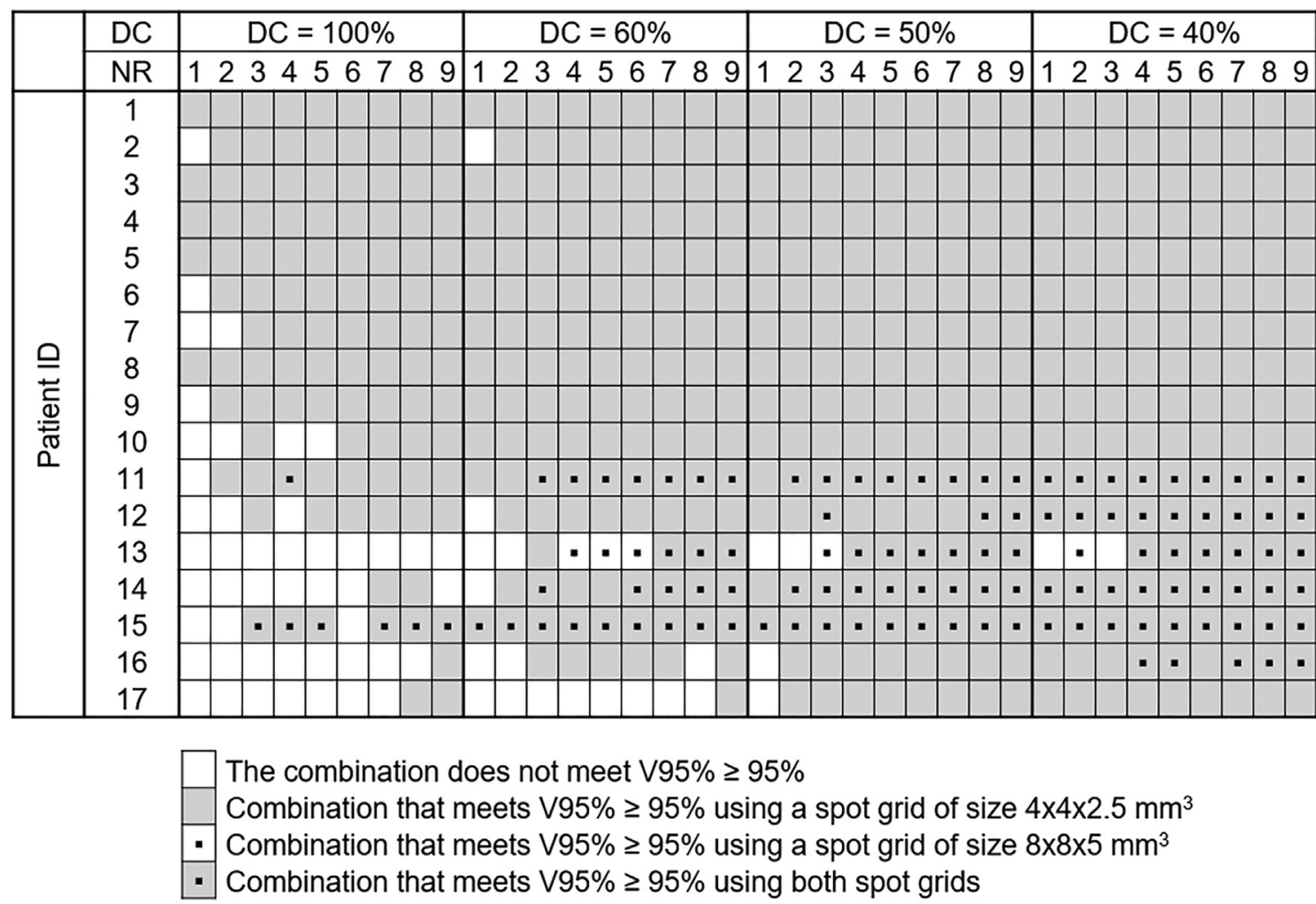

(b)

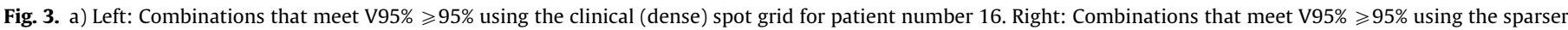

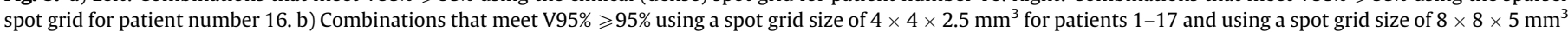
for patients 11-16.Abbreviations: DC: duty cycle; NR: number of rescanning.

reduced up to $12 \%$ for the patient with the largest CTV SI motion using a $40 \%$ DC and the clinical spot grid.

It has also been shown that the spot grid size influences the results. Although the time needed to deliver the same plan is slightly reduced using the sparser spot grid (less spots), the denser spot grid gave better CTV dose coverage for all plans. By this means, except for one patient, the most time efficient plan using the $4 \times 4 \times 2.5 \mathrm{~mm}^{3}$ spot grid would even not be acceptable with the $8 \times 8 \times 5 \mathrm{~mm}^{3}$ spot grid.
Even though our results are only valid for this patient cohort and for a fast energy switching delivery machine, these results can be taken as a starting point for PBS based treatment of future patients with thoracic and abdominal tumors. The 4DDC will still be required in all future cases. A more extensive investigation could also be conducted when considering the extent of motion of the CTV as a definition of the gating window. Another limitation of this study is that we relied on a single $4 \mathrm{DCT}$ per patient, which assumes that the patient breathes the same way during the treat- 
Table 3

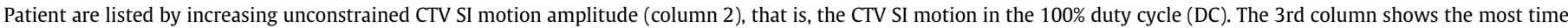

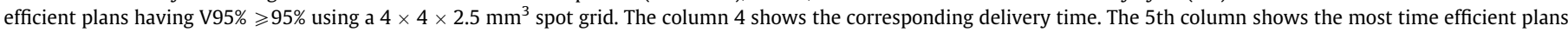

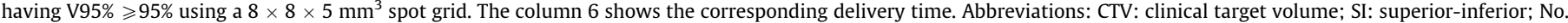
fields: number of fields; DC: duty cycle; NR: number of rescanning.

\begin{tabular}{|c|c|c|c|c|c|c|}
\hline Patient ID & CTV SI motion (mm) & $4 \times 4 \times 2.5 \mathrm{~mm}^{3}$ & Time (min) & $8 \times 8 \times 5 \mathrm{~mm}^{3}$ & Time (min) & No. fields \\
\hline 1 & 1.87 & $\mathrm{DC}=100 \% \mathrm{NR}=1$ & 1.6 & & & 2 \\
\hline 2 & 2 & $\mathrm{DC}=100 \% \mathrm{NR}=2$ & 9.7 & & & 2 \\
\hline 3 & 2.45 & $\mathrm{DC}=100 \% \mathrm{NR}=1$ & 1.4 & & & 1 \\
\hline 4 & 2.45 & $\mathrm{DC}=100 \% \mathrm{NR}=1$ & 4.8 & & & 3 \\
\hline 5 & 4.53 & $\mathrm{DC}=100 \% \mathrm{NR}=1$ & 7.0 & & & 2 \\
\hline 6 & 5.57 & $\mathrm{DC}=100 \% \mathrm{NR}=2$ & 2.0 & & & 3 \\
\hline 7 & 5.69 & $\mathrm{DC}=100 \% \mathrm{NR}=3$ & 1.7 & & & 2 \\
\hline 8 & 6.59 & $\mathrm{DC}=100 \% \mathrm{NR}=1$ & 2.3 & & & 3 \\
\hline 9 & 6.64 & $\mathrm{DC}=100 \% \mathrm{NR}=2$ & 1.6 & & & 3 \\
\hline 10 & 7.73 & $\mathrm{DC}=100 \% \mathrm{NR}=3$ & 1.7 & & & 3 \\
\hline \multirow[t]{4}{*}{11} & 8.44 & $\mathrm{DC}=100 \% \mathrm{NR}=2$ & 2.1 & $\mathrm{DC}=100 \% \mathrm{NR}=4$ & 2.3 & 3 \\
\hline & & & & $\mathrm{DC}=40 \% \mathrm{NR}=1$ & 3.1 & \\
\hline & & & & $\mathrm{DC}=50 \% \mathrm{NR}=2$ & 3.2 & \\
\hline & & & & $\mathrm{DC}=60 \% \mathrm{NR}=3$ & 3.2 & \\
\hline \multirow[t]{3}{*}{12} & 11.36 & $\mathrm{DC}=100 \% \mathrm{NR}=3$ & 3.3 & $\mathrm{DC}=40 \% \mathrm{NR}=1$ & 3.7 & 3 \\
\hline & & $\mathrm{DC}=50 \% \mathrm{NR}=1$ & 3.9 & & & \\
\hline & & $\mathrm{DC}=60 \% \mathrm{NR}=2$ & 4.2 & & & \\
\hline \multirow[t]{3}{*}{13} & 12.14 & $\mathrm{DC}=60 \% \mathrm{NR}=3$ & 1.8 & $\mathrm{DC}=60 \% \mathrm{NR}=4$ & 1.8 & 2 \\
\hline & & $\mathrm{DC}=50 \% \mathrm{NR}=5$ & 2.4 & $\mathrm{DC}=40 \% \mathrm{NR}=2$ & 1.9 & \\
\hline & & & & $\mathrm{DC}=50 \% \mathrm{NR}=3$ & 1.9 & \\
\hline \multirow[t]{3}{*}{14} & 12.95 & $\mathrm{DC}=50 \% \mathrm{NR}=1$ & 3.9 & $\mathrm{DC}=60 \% \mathrm{NR}=3$ & 3.4 & 2 \\
\hline & & $\mathrm{DC}=60 \% \mathrm{NR}=2$ & 4.7 & $\mathrm{DC}=50 \% \mathrm{NR}=2$ & 3.5 & \\
\hline & & $\mathrm{DC}=40 \% \mathrm{NR}=1$ & 4.9 & & & \\
\hline \multirow[t]{2}{*}{15} & 14.76 & $\mathrm{DC}=100 \% \mathrm{NR}=3$ & 3.5 & $\mathrm{DC}=100 \% \mathrm{NR}=3$ & 2.8 & 2 \\
\hline & & $\mathrm{DC}=60 \% \mathrm{NR}=1$ & 3.6 & & & \\
\hline \multirow[t]{3}{*}{16} & 21.79 & $\mathrm{DC}=60 \% \mathrm{NR}=3$ & 5.9 & & & 3 \\
\hline & & $\mathrm{DC}=50 \% \mathrm{NR}=2$ & 5.9 & $\mathrm{DC}=40 \% \mathrm{NR}=4$ & 7.8 & \\
\hline & & $\mathrm{DC}=40 \% \mathrm{NR}=1$ & 6.1 & & & \\
\hline \multirow[t]{3}{*}{17} & 30.07 & $\mathrm{DC}=50 \% \mathrm{NR}=2$ & 3.2 & & & 2 \\
\hline & & $\mathrm{DC}=100 \% \mathrm{NR}=8$ & 3.7 & & & \\
\hline & & $\mathrm{DC}=40 \% \mathrm{NR}=1$ & 3.7 & & & \\
\hline
\end{tabular}

ment delivery, and ignored any possible variation in motion, whether intra- or inter-fractional. However, respiratory patterns vary from one fraction to another and even within a fraction, especially in the case of free breathing. That is why coaching could be applied to improve the reproducibility of respiration using external surrogates to monitor breathing [50]. Last but not least, we did not simulate any fractionation, this would, however, act as an additional 'rescanning' [51], and thus over the whole treatment, more rescanning/gating combinations might become clinically acceptable.

\section{Appendix A. Supplementary data}

Supplementary data associated with this article can be found, in the online version, athttps://doi.org/10.1016/j.radonc.2021.03.041.

\section{References}

[1] Phillips MH, Pedroni E, Blattmann H, Boehringer T, Coray A, Scheib S. Effects of respiratory motion on dose uniformity with a charged particle scanning method. Phys Med Biol 1992;37:223-34. https://doi.org/10.1088/0031-9155/ 37/1/016. ISSN 00319155.

[2] Bert C, Durante M. Motion in radiotherapy: Particle therapy. Phys Med Biol 2011;56. https://doi.org/10.1088/0031-9155/56/16/R01. ISSN 00319155.

[3] Zenklusen SM, Pedroni E, Meer D. A study on repainting strategies for treating moderately moving targets with proton pencil beam scanning at the new gantry 2 at PSI. Phys Med Biol 2010;55:5103-21. https://doi.org/10.1088/ 0031-9155/55/17/014. ISSN 00319155.

[4] Keall Paul J, Mageras Gig S, Balter James M, Emery Richard S, Forster Kenneth $\mathrm{M}$, Jiang Steve B, et al. The management of respiratory motion in radiation oncology report of AAPM Task Group 76. Med Phys 2006;33:3874-900. https://doi.org/10.1118/1.2349696. ISSN 00942405.

[5] Grözinger Sven Oliver, Bert Christoph, Haberer Thomas, Kraft Gerhard, Rietzel Eike. Motion compensation with a scanned ion beam: A technical feasibility study. Radiation Oncol 2008;3:1-7. https://doi.org/10.1186/1748-717X-3-34. ISSN 1748717X

[6] Knopf Antje, Bert Christoph, Heath Emily, Nill Simeon, Kraus Kim, Richter Daniel. Special report: Workshop on 4D-treatment planning in actively scanned particle therapy-Recommendations, technical challenges, and future research directions. Med Phys 2010;37:4608-14. https://doi.org/10.1118/ 1.3475944. ISSN 00942405

[7] Rietzel Eike, Bert Christoph. Respiratory motion management in particle therapy. Med Phys 2010;37:449-60. https://doi.org/10.1118/1.3250856. ISSN 00942405.

[8] Wong Victy YW, Tung Stewart Y, Ng Alice WY, Li Francis AS, Leung Joyce OY. Real-time monitoring and control on deep inspiration breath-hold for lung cancer radiotherapy-Combination of $\mathrm{ABC}$ and external marker tracking. Med Phys 2010;37:4673-83. https://doi.org/10.1118/1.3476463. ISSN 00942405.

[9] Bert Christoph, Gemmel Alexander, Saito Nami, Rietzel Eike. Gated Irradiation With Scanned Particle Beams. Int J Rad Oncol Biol Phys 2009;73:1270-5. https://doi.org/10.1016/i.iirobp.2008.11.014. ISSN 03603016.

[10] Schätti A, Zakova M, Meer D, Lomax AJ. The effectiveness of combined gating and re-scanning for treating mobile targets with proton spot scanning. An experimental and simulation-based investigation. Phys Med Biol 2014;59:3813-28. https://doi.org/10.1088/0031-9155/59/14/3813. ISSN 13616560.

[11] Furukawa Takuji, Inaniwa Taku, Sato Shinji, Shirai Toshiyuki, Mori Shinichiro, Takeshita Eri, et al. Moving target irradiation with fast rescanning and gating in particle therapy. Med Phys 2010;37:4874-9. https://doi.org/10.1118/ 1.3481512. ISSN 00942405.

[12] Keall PJ, Kini VR, Vedam SS, Mohan R. Potential radiotherapy improvements with respiratory gating. Australasian Phys Eng Sci Med 2002;25:1-6. https:// doi.org/10.1007/BF03178368. ISSN 01589938.

[13] Bert Christoph, Saito Nami, Schmidt Alexander, Chaudhri Naved, Schardt Dieter, Rietzel Eike. Target motion tracking with a scanned particle beam. Med Phys 2007;34:4768-71. https://doi.org/10.1118/1.2815934@10.1002/(ISSN) 2473-4209.MEDICALPHYSICSLETTER. ISSN 2473-4209.

[14] Lüchtenborg Robert, Saito Nami, Durante Marco, Bert Christoph. Experimental verification of a real-time compensation functionality for dose changes due to target motion in scanned particle therapy. Med Phys 2011;38:5448-58. https://doi.org/10.1118/1.3633891. ISSN 00942405.

[15] Zhang Ye, Knopf A, Tanner C, Lomax AJ. Online image guided tumour tracking with scanned proton beams: A comprehensive simulation study. Physics in Medicine and Biology 2014;59:7793-817. https://doi.org/10.1088/0031-9155/ 59/24/7793. 10.1088/0031-9155/59/24/7793. 
[16] Zhang Ye, Huth Isabel, Wegner Martin, Charles Weber Damien, Lomax Antony John. An evaluation of rescanning technique for liver tumour treatments using a commercial PBS proton therapy system. Radiotherapy Oncol 2016;121:281-7. https://doi.org/10.1016/i.radonc.2016.09.011. ISSN 18790887.

[17] Bernatowicz K, Lomax AJ, Knopf A. Comparative study of layered and volumetric rescanning for different scanning speeds of proton beam in liver patients. Phys Med Biol 2013;58:7905-20. https://doi.org/10.1088/00319155/58/22/7905. ISSN 13616560.

[18] Grassberger Clemens, Dowdell Stephen, Lomax Antony, Sharp Greg, Shackleford James, Choi Noah, et al. Motion interplay as a function of patient parameters and spot size in spot scanning proton therapy for lung cancer. International Journal of Radiation Oncology Biology Physics 2013;86:380-6. https://doi.org/10.1016/j.ijrobp.2013.01.024. ISSN 03603016.

[19] Li Yupeng, Kardar Laleh, Li Xiaoqiang, Li Heng, Cao Wenhua, Chang Joe Y, et al. On the interplay effects with proton scanning beams in stage III lung cancer. Med Phys 2014;41:021721. https://doi.org/10.1118/1.4862076. ISSN 00942405.

[20] Schätti A, Zakova M, Meer D, Lomax AJ. Experimental verification of motion mitigation of discrete proton spot scanning by re-scanning. Phys Med Biol 2013;58:8555-72. https://doi.org/10.1088/0031-9155/58/23/8555. ISSN 13616560.

[21] Bert Christoph, Grözinger Sven O, Rietzel Eike. Quantification of interplay effects of scanned particle beams and moving targets. Phys Med Biol 2008;53:2253-65. https://doi.org/10.1088/0031-9155/53/9/003. ISSN 00319155.

[22] Knopf Antje Christin, Boye Dirk, Lomax Antony, Mori Shininchiro. Adequate margin definition for scanned particle therapy in the incidence of intrafractional motion. Phys Med Biol 2013;58:6079-94. https://doi.org/ 10.1088/0031-9155/58/17/6079. ISSN 00319155.

[23] Giovanni Fattori, Jan Hrbacek, Harald Regele, Christian Bula, Alexandre Mayor, Stefan Danuser, David C. Oxley, Urs Rechsteiner, Martin Grossmann, Riccardo Via, Till T. Böhlen, Alessandra Bolsi, Marc Walser, Michele Togno, Emma Colvill, Daniel Lempen, Damien C. Weber, Antony J. Lomax, and Sairos Safai. Commissioning and Quality Assurance of a novel solution for respiratorygated PBS proton therapy based on optical tracking of surface markers. Zeitschrift fur Medizinische Physik, pages 1-11, 2020. ISSN 18764436. DOI: 10.1016/j.zemedi.2020.07.001. doi: 10.1016/j.zemedi.2020.07.001.

[24] Vedam SS, Keall PJ, Kini VR, Mostafavi H, Shukla HP, Mohan R. Acquiring a four-dimensional computed tomography dataset using an external respiratory signal. Phys Med Biol 2003;48:45-62. https://doi.org/10.1088/0031-9155/48/ 1/304. ISSN 00319155.

[25] Engelsman Martijn, Rietzel Eike, Kooy Hanne M. Four-dimensional proton treatment planning for lung tumors. Int J Radiation Oncol Biol Phys 2006;64:1589-95. https://doi.org/10.1016/j.ijrobp.2005.12.026.

[26] Keall Paul. 4-Dimensional Computed Tomography Imaging and Treatment Planning. Seminars Radiation Oncol 2004;14:81-90. https://doi.org/10.1053/i. semradonc.2003.10.006. ISSN 10534296.

[27] Pan Tinsu, Yim Lee Ting, Rietzel Eike, Chen George TY. 4D-CT imaging of a volume influenced by respiratory motion on multi-slice CT. Med Phys 2004;31:333-40. https://doi.org/10.1118/1.1639993. ISSN 00942405.

[28] Rietzel Eike, Chen George TY, Choi Noah C, Willet Christopher G. Fourdimensional image-based treatment planning: Target volume segmentation and dose calculation in the presence of respiratory motion. Int J Radiation Oncol Biol Phys 2005;61:1535-50. https://doi.org/10.1016/j. iirobp.2004.11.037.

[29] Rueckert D, Sonoda LI, Hayes C, Hill DLG, Leach MO, Hawkes DJ. Nonrigid registration using free-form deformations: application to breast MR images. IEEE Trans Med Imaging 1999;18(8):712-21.

[30] Hajnal Joseph V, Hill Derek LG, Hawkes David J. Medical image registration. Med Image Registration 2001:1-383. https://doi.org/10.1051/epn:2000401.

[31] Rietzel Eike, Chen George TY. Deformable registration of 4D computed tomography data. Med Phys 2006;33:4423-30. https://doi.org/10.1118/ 1.2361077

[32] Bernatowicz Kinga, Peroni Marta, Perrin Rosalind, Weber Damien C, Lomax Antony. Four-Dimensional Dose Reconstruction for Scanned Proton Therapy Using Liver 4DCT-MRI. Int J Radiation Oncology Biol Phys 2016;95(1):216-23.

[33] Kirby Neil, Chuang Cynthia, Ueda Utako, Pouliot Jean. The need for applicationbased adaptation of deformable image registration. Med Phys 2013;40. https://doi.org/10.1118/1.4769114. ISSN 00942405.
[34] Ribeiro Cássia O, Knopf Antje, Langendijk Johannes A, Weber Damien C, Lomax Antony J, Zhang Ye. Assessment of dosimetric errors induced by deformable image registration methods in $4 \mathrm{D}$ pencil beam scanned proton treatment planning for liver tumours. Radiotherapy Oncol 2018;128:174-81. https://doi. org/10.1016/i.radonc.2018.03.001. ISSN 18790887.

[35] Balter James M, Lam Kwok L, McGinn Cornealeus J, Lawrence Theodore S, Ten Haken Randall K. Improvement of CT-based treatment-planning models of abdominal targets using static exhale imaging. International Journal of Radiation Oncology Biol Phys 1998;41:939-43. https://doi.org/10.1016/ S0360-3016(98)00130-8. ISSN 03603016.

[36] Ritchie CJ, Hsieh J, Gard MF, Godwin JD, Kim Y, Crawford CR. Predictive respiratory gating: a new method to reduce motion artifacts on CT scans. Radiology 1994;190.

[37] Pedroni E, A novel gantry for proton therapy at the Paul Scherrer Institute. 13 (February):13-17, 2003. DOI: 10.1063/1.1435184..

[38] Knopf Antje Christin, Hong Theodore S, Lomax Antony. Scanned proton radiotherapy for mobile targets - The effectiveness of re-scanning in the context of different treatment planning approaches and for different motion characteristics. Phys Med Biol 2011;56:7257-71. https://doi.org/10.1088/ 0031-9155/56/22/016. ISSN 00319155.

[39] Inaniwa Taku, Furukawa Takuji, Tomitani Takehiro, Sato Shinji, Noda Koji, Kanai Tatsuaki. Optimization for fast-scanning irradiation in particle therapy. Med Phys 2007;34:3302-11. https://doi.org/10.1118/1.2754058. ISSN 00942405.

[40] Pedroni Eros, Bearpark Ralph, Böhringer Terence, Coray Adolf, Duppich Jürgen. Sven Forss, et al. The PSI Gantry 2: A second generation proton scanning gantry. Zeitschrift fur Medizinische Physik 2004;14:25-34. https://doi.org/ 10.1078/0939-3889-00194. ISSN 09393889.

[41] Pedroni E, Meer D, Bula C, Safai S, Zenklusen S. Pencil beam characteristics of the next-generation proton scanning gantry of PSI: Design issues and initial commissioning results. Eur Phys J Plus 2011;126:1-27. https://doi.org/ 10.1140/epjp/i2011-11066-0. ISSN 21905444.

[42] Safai Sairos, Bula Christian, Meer David, Pedroni Eros. Improving the precision and performance of proton pencil beam scanning. Transl Cancer Res 2012;1:196-206. https://doi.org/10.3978/j.issn.2218-676X.2012.10.08. ISSN 22196803.

[43] ICRU. Prescribing, recording and reporting photon beam therapy (supplement to ICRU report 50). Technical report, Bethesda, USA, 1999.

[44] Rietzel Eike, Liu Arthur K, Doppke Karen P, Wolfgang John A, Chen Aileen B, Chen George TY, Choi Noah C. Design of 4D treatment planning target volumes. International Journal of Radiation Oncology Biology Physics 2006;66:287-95. https://doi.org/10.1016/j.ijrobp.2006.05.024. ISSN 03603016.

[45] Kang Yixiu, Zhang Xiaodong, Chang Joe Y, Wang He, Wei Xiong, Liao Zhongxing, et al. 4D Proton treatment planning strategy for mobile lung tumors. Int J Radiation Oncol Biol Phys 2007;67:906-14. https://doi.org/ 10.1016/i.iirobp.2006.10.045. ISSN 03603016.

[46] Botas Pablo, Grassberger Clemens, Sharp Gregory, Paganetti Harald. Density overwrites of internal tumor volumes in intensity modulated proton therapy plans for mobile lung tumors. Phys Med Biol 2018;63. https://doi.org/10.1088/ 1361-6560/aaa035. ISSN 13616560.

[47] Lomax A. SFUD, IMPT, and Plan Robustness. In Particle Radiotherapy, pages 169-194. 2016..

[48] Boye Dirk, Lomax Tony, Knopf Antje. Mapping motion from 4D-MRI to 3D-CT for use in 4D dose calculations: A technical feasibility study. Med Phys 2013;40. https://doi.org/10.1118/1.4801914. ISSN 00942405.

[49] Miriam Krieger, Grischa Klimpki, Giovanni Fattori, Jan Hrbacek, David Oxley, Sairos Safai, Damien C. Weber, Antony J. Lomax, and Ye Zhang. Experimental validation of a deforming grid $4 \mathrm{D}$ dose calculation for PBS proton therapy. Phys Med Biol, 63(5), 2018. ISSN 13616560. DOI: 10.1088/1361-6560/aaad1e..

[50] Edward D. Brandner, Indrin J. Chetty, Tawfik G. Giaddui, Ying Xiao, and M. Saiful Huq. Motion Management Strategies and Technical Issues Associated with Stereotactic Body Radiotherapy of Thoracic and Upper Abdominal. Physiology \& behavior, 176(3):139-148, 2017. 10.1016/ j.physbeh.2017.03.040.

[51] Kraus KM, Heath E, Oelfke U. Dosimetric consequences of tumour motion due to respiration for a scanned proton beam. Phys Med Biol 2011;56:6563-81. https://doi.org/10.1088/0031-9155/56/20/003. ISSN 00319155. 